\title{
Gentiopicroside promotes osteogenesis and prevents bone loss in ovariectomized mice by modulation of $\beta$-catenin-BMP2 signaling pathway
}

Huaji Jiang

Shantou University Medical College

Jialiang Zhong

Sun Yat-Sen University

Wenjun Li

Shantou University Medical College

Jianghui Dong

University of South Australia

Cory J Xian

University of South Australia

Yung-Kang Shen

Taipei Medical University

Lufeng Yao

Ningbo No 6 Hospital

Qiang Wu

Shantou University Medical College

Liping Wang ( $\sim$ liping.wang@mymail.unisa.edu.au )

University of South Australia https://orcid.org/0000-0001-9355-1167

\section{Research}

Keywords: Gentiopicroside, osteoporosis, Wnt/ $\beta$-catenin pathway, BMP pathway, osteogenic factors, osteogenic parameters

Posted Date: August 3rd, 2020

DOI: https://doi.org/10.21203/rs.3.rs-50702/v1

License: (c) (1) This work is licensed under a Creative Commons Attribution 4.0 International License.

Read Full License 


\section{Abstract}

Background: Osteoporosis is characterized by increased bone fragility and the drugs used at present to treat osteoporosis can cause adverse reactions. Gentiopicroside (GEN) is widely used in the traditional Chinese medicine thanks to its multiple pharmacological activities including the suppression of osteoclastogenesis. Therefore, the aim of this work was to explore the effect of GEN on bone mesenchymal stem cells (BMSCs) osteogenesis for a potential osteoporosis therapy.

Methods: In vitro, BMSCs were exposed to GEN at different doses for 2 weeks, while in vivo, ovariectomized osteoporosis was established in mice and the therapeutic effect of GEN was evaluated for 3 months.

Results: Our results in vitro showed that GEN promoted the activity of alkaline phosphatase, increased the calcified nodules in BMSCs and upregulated the osteogenic factors (Runx2, OSX, OCN, OPN, and BMP2). In vivo, GEN strengthened the secretion of Runx2, OCN, and BMP2, increased the level of osteogenic parameters, and accelerated the osteogenesis of BMSCs by activating the BMP pathway and Wnt/ $\beta$ catenin pathway, effect that was inhibited using the BMP inhibitor Noggin and Wnt/ $\beta$-catenin inhibitor DKK1. Silencing the $\beta$-catenin gene and BMP2 gene blocked the osteogenic differentiation induced by GEN in BMSCs. This block was also observed when only $\beta$-catenin was silenced, while the knockout of BMP2 did not affect $\beta$-catenin expression induced by GEN. Interpretation.

Conclusions: GEN promotes BMSC osteogenesis by regulating $\beta$-catenin-BMP signalling, providing a novel strategy in the treatment of osteoporosis.

\section{Background}

Osteoporosis is a systemic disease characterized by bone loss, destruction of the bone microstructure and increased bone fragility, often leading to brittle fractures [1]. A large number of patients with osteoporosis not only suffer from severe pain, but they are also subjected to a heavy financial burden [2]. In particular, the bone resorption rate of postmenopausal women is significantly higher than that of osteogenesis, leading to a serious bone loss [3]. With the development of aging population, the number of postmenopausal patients with osteoporosis is increasing year by year, causing a significant impact on the medical community and the whole society [4]. At present, the drugs used to treat postmenopausal osteoporosis play a role mainly by inhibiting bone absorption and promoting bone formation $[5,6]$. However, long-term use of anti-osteoporosis drugs can cause a series of adverse reactions, including myasthenia gravis, influenza like diseases, and gastrointestinal tumours [7]. Thus, it is of utmost importance to find a new alternative therapy to cure osteoporosis. The activity of osteoclasts and osteoblasts needs to be precisely coordinated to maintain skeletal integrity [8]. Osteoclasts are multinucleated giant cells derived from monocytes/macrophages, whose main function is to promote bone resorption [9]. Osteoblasts are mainly differentiated from bone mesenchymal stem cells (BMSCs) and deposited in the calcified bone matrix, which has a significant impact on the formation of a new 
bone [10]. The primary cause of osteoporosis is due to the decrease in osteoblasts that leads to a reduced bone formation, and the increase in osteoclasts resulting in an increased osteolysis. Recently, several reports pointed out that stimulating osteoblast differentiation may be an effective way to prevent and treat osteoporosis [11-13].

BMSCs are stem cells with a multi-directional differentiation, since they can differentiate into several cell types including chondrocytes, osteoblasts, adipocytes, and endothelial cells under specific conditions [14]. BMSCs first differentiate into precursor osteoblasts, then into osteoblasts, and finally they gradually form the mature osteoblasts [15].However, the osteogenic capability of BMSCs gradually decreases with the increase of old age, while the adipogenic capacity of BMSCs increased, leading to the downregulation of bone formation and finally osteoporosis [16]. During the osteogenic differentiation, BMSCs release a series of osteogenic factors, including osteocalcin (OCN), osterix (OSX), osteopontin (OPN), and runtrelated transcription factor 2 (Runx2), which accelerate the maturation of osteoblasts $[16,17]$. Therefore, BMSCs may be a suitable cell source for studying osteogenesis.

The Wnt pathway and BMP pathway occupy a critical position in the modulation of osteoblast differentiation $[18,19]$. When the frizzled transmembrane receptor binds to LRP5 and/or LRP6, it can induce the secretion of Wnts and activates the canonical Wnt pathway [20]. Subsequently, $\beta$-catenin is released and transcribed into the nucleus to regulate the generation of osteogenic markers [21]. The upregulation of BMP pathway induces the phosphorylation of Smad proteins [22]. Then, the activated Smad proteins actively regulate the transcription of osteogenic factors (Runx2 and OCN), thus promoting osteogenic differentiation $[23,24]$.

Gentiopicroside (GEN) is the active component of the plant Gentiana Manshurica Kitag, widely used in the traditional Chinese medicine thanks to its multiple pharmacological activities, such as antioxidative, antinociceptive, anti-inflammatory, antibacterial, and antiosteoporotic effects [25-27]. Osteoblast differentiation and osteoclastogenesis are two important phases of bone remodelling $[28,29]$ and GEN represents a potential drug in the treatment of osteoporosis by suppressing osteoclastogenesis [30]. Based on the above studies, our hypothesis is that GEN could induce osteogenic differentiation of BMSCs in vitro and bone formation in vivo.

Therefore, the purpose of this study was to find whether GEN could promote the osteogenic differentiation of BMSCs and explore the molecular mechanism induced by GEN in differentiating BMSCs.

\section{Results}

\section{Effect of GEN on BMSC proliferation}

The chemical structure of GEN is shown in Fig. 1a. The results suggested that the proliferation of BMSCs treated with GEN $(10-40 \mu \mathrm{M})$ was not significantly changed. However, $80 \mu \mathrm{M}$ GEN significantly inhibited 
the proliferation of BMSCs (Fig. 1b). Thus, GEN was not harmful to BMSCs at the concentrations of 10, 20 , and $40 \mu \mathrm{M}$.

\section{GEN strengthens the osteogenic differentiation in BMSCs}

The effect of GEN alone on BMSCs was tested as first. The results showed that the osteogenic differentiation of the BMSCs treated with GEN for 14 days without osteogenic medium was not significantly affected (Supplementary Fig. 1). Next, the osteogenic differentiation of BMSCs treated with GEN for two weeks under osteogenic induction was explored. The results showed that the higher concentration of GEN, the higher ALP activity (Fig. 1c, e) and the number of mineralized nodules (Fig. 1d, f) compared with the control group. In addition, the mRNA expression of the osteogenic genes (ALP, Runx2, OSX, OCN, OPN, and BMP2) was significantly increased by GEN, and the increasing trend was concentration-dependent (Fig. 2a-f). Similarly, the expression of osteogenic proteins (Runx2, OCN, and BMP2) were also promoted by GEN and reached a peak at $40 \mu \mathrm{M}$ (Fig. 3a-d). Therefore, our results revealed that GEN strengthened the osteogenic differentiation in BMSCs.

\section{GEN promotes bone formation in OVX mice}

The OVX mouse model was used to confirm these in vitro results. The OVX osteoporosis mouse model is the most commonly used animal model in studying postmenopausal osteoporosis. Ovariectomy can cause bone loss acceleration and cortical bone formation reduction, which are closely related to oestrogen deficiency $[31,29]$. To test the effect of the ovariectomy, the body weight and the mass of the uterus were measured (Supplementary Fig. 2). The results showed that the body weight of the OVX group and OVX + GEN group was greater than that of the Sham group (Supplementary Fig. 2a). In contrast, the mass of the uterus in the OVX group and OVX + GEN group was less than that in the Sham group (Supplementary Fig. 2b). The histopathological images of all groups (sham group, OVX group, and OVX+ GEN group) are shown in Fig. 4. HE staining results showed that the number of bone trabeculae in the OVX group was significantly less than that in the Sham group, whereas the number of bone trabeculae in the OVX + GEN group was higher than that in the OVX group, but no significant difference was observed between the Sham group and OVX + GEN group (Fig. 4a). The results of micro-CT showed that the BMD, the number of trabeculae and the thickness of the trabeculae in OVX + GEN group were higher than those in the OVX group, while the same parameters in the OVX group were remarkably lower than those in the Sham group (Fig. 4b-e). However, no statistical difference was found between OVX + GEN group and Sham group.

Subsequently, immunohistochemistry was used to detect the secretion of osteogenic proteins (Runx2, OCN, and BMP2) in vivo. Runx2 expression in the OVX group was less than that in the Sham group, while its expression in the OVX + GEN group was higher than that in the OVX group (Fig. 5a). Similar to the results of Runx2, the expression of OCN (Fig. 5b, e) and BMP2 (Fig. 5c, f) in OVX group was lower than that in the Sham group and OVX + GEN group. Thus, our results demonstrate that GEN effectively promoted osteogenesis in OVX osteoporotic mice and showed a good anti-osteoporotic effect. 


\section{BMP pathway and Wnt/ $\beta$-catenin pathway activated by GEN in BMSCs}

Since the BMP signalling [32] and WNT signalling [33] pathways are related to osteogenesis, the consequence of GEN treatment on the signalling of BMP and WNT/ $\beta$-catenin was evaluated. Without osteogenic induction, GEN did not alter the expression of $p$-Smad1/5/8 and $\beta$-catenin in BMSCs (Supplementary Fig. 3). Next, the effect of GEN on the osteogenic mechanism in BMSCs was further explored. After the treatment of BMSCs with GEN for 2 weeks under osteogenic conditions, the results showed that GEN upregulated the expression of $p$-Smad1/5/8 and $\beta$-catenin in a dose-dependent manner (Fig. 6a, b, c). However, the osteogenesis-potentiating effect of GEN on p-Smad1/5/8 and $\beta$-catenin was abolished by the treatment with the BMP pathway inhibitor Noggin (Fig. 7a-c) and the Wnt/ $\beta$-catenin pathway inhibitor DKK1 (Fig. 7d-f). Therefore, our results reveal that GEN promoted the osteogenic differentiation via BMP signalling and WNT/ $\beta$-catenin signalling.

\section{GEN-induced osteogenic differentiation is a $\beta$-catenin- BMP2-dependent effect}

To further reveal the specific mechanism of GEN in regulating of BMP signalling and Wnt/ $\beta$-catenin signalling, gene silencing was performed in vitro. The transfection with Ad-Cre efficiently silenced the $\beta$ catenin (Fig. 8a) and BMP2 (Fig. 8g) gene in BMSCs. $\beta$-catenin silencing in BMSCs significantly inhibited the increase of RunX2, OSX,OCN,OPN, and BMP2 induced by GEN (Fig. 8b-f). In addition, the increase of Runx2, OSX, OCN, and OPN (Fig. 8h-k) induced by GEN was inhibited by silencing the BMP2 gene, while the expression of $\beta$-catenin was not affected (Fig. 8I). Our data revealed that the silencing of $\beta$-catenin gene prevented GEN-mediated upregulation of BMP2, although GEN-induced $\beta$-catenin enhancement was not influenced by silencing the BMP2 gene. The above results indicated that GEN first activates the $\beta$ catenin pathway and then the BMP2 pathway. Therefore, GEN strengthened the osteogenic ability of BMSCs through the $\beta$-catenin-BMP2 signalling pathway.

\section{Discussion}

GEN is considered an effective drug in the treatment of osteoporosis by preventing the formation of osteoclast [30]. The inhibition of osteoclast or the activation of osteogenesis exerts a significantly protective effect on osteoporosis [34]. The current study demonstrated for the first time the effect and potential mechanism of GEN in the osteogenic process of BMSCs both in vitro and in vivo. BMSCs were used to explore the effect of GEN in osteogenesis in vitro. GEN did not show any toxicity on BMSCs at the doses used. In addition, GEN enhanced the expression of ALP and improved the mineralized nodules, increased the expression of osteogenic factors in a concentration dependent manner. Finally, the in vivo results showed that GEN accelerated the osteoid formation and mineralization in the mouse femur. All these results indicated that GEN could stimulate osteogenesis in vitro and in vivo. Our results showed that GEN strengthened the osteogenic ability of BMSCs in vitro and stimulated bone ossification in vivo 
by the upregulation of the $\beta$-catenin-BMP2 signalling pathway. Therefore, GEN could be potentially considered a novel compound in regulating bone metabolism, since it could not only promote osteogenesis but also inhibit bone absorption.

BMP pathway and Wnt pathway occupy an critical position in determining the direction of osteogenesis in BMSCs $[35,36]$. The activation of the BMP pathway induces the phosphorylation of Smad1/5/8. Then, the phosphorylated Smad1/5/8 bind to Smad4 and move into the nucleus, thus activating downstream factors of the BMP pathway [37]. Additionally, the Wnt/ $\beta$-catenin signalling is critical in the therapy of osteoporosis, since it has a significant impact on promoting osteogenesis and regulating bone metabolism [38]. When the Wnt/ $\beta$-catenin signalling is upregulated, it can effectively promote the transformation of the precursor osteoblasts into osteoblasts, so as to actively regulate the formation of new bone and improve the structure of the bone itself [39]. After Wnt pathway activation, the $\beta$-catenin is efficiently transcribed into the nucleus, thus stimulating the production of downstream factors [40]. Our results revealed that GEN activated both the BMP pathway and Wnt/ $\beta$-catenin pathway in BMSCs. It also demonstrated that Noggin [41], and DKK1 [42], significantly inhibited GEN-induced osteogenesis in BMSCs. Therefore, GEN stimulated the osteogenic ability of BMSCs through both the two signalling pathways.

To reveal the sequential order in the activation of the BMP pathway and Wnt pathway by GEN, $\beta$-catenin or BMP2 were silenced in vitro, resulting in a suppression of GEN-mediated osteogenesis after the silencing of both genes. In addition, the silencing of $\beta$-catenin completely suppressed GEN-mediated BMP2 expression, whereas the silencing of BMP2 could not influence GEN-mediated $\beta$-catenin expression in BMSCs. Thus, BMP2 was a target factor in the downstream of the $\beta$-catenin pathway in BMSCs. Overall, our results demonstrated that GEN stimulated the ossification of BMSCs by the activation of $\beta$ catenin-BMP2 pathway.

Taken together the results in the current study, a model illustrating the potential mechanism used by GEN to promote the osteogenic differentiation of BMSCs could be proposed. GEN stimulates osteogenesis by increasing ALP, Runx2, OSX, OCN, and OPN though the activation of the Wnt/ $\beta$-catenin-BMP2 signalling, thereby promoting the differentiation of BMSCs into osteoblasts (Fig. 9).

\section{Conclusions}

In conclusion, this study provided novel insights into the effect of GEN on BMSCs osteogenic differentiation and its protective effect against bone loss. Although further studies are required to confirm these results, GEN might represent a promising approach in the treatment of osteoporosis.

\section{Materials And Methods}

This project was carried out with the permission of the Ethics Committee of the Ningbo No. 6 Hospital (registered number 2015-018). 


\section{Cell culture and treatments}

Five female C57BL/ 6 mice (4-week-old) were used and euthanized to obtain BMSCs cultured according to a previous work [43]. In brief, the mouse femur was collected and the surrounding soft tissues were removed. Then, the bone marrow in the femur was washed three times with a-MEM (Sigma-Aldrich). The obtained bone marrow content was placed in a dish containing a complete medium consisting of a medium supplemented with $100 \mathrm{U} / \mathrm{ml}$ penicillin, $100 \mathrm{mg} / \mathrm{ml}$ streptomycin sulphate, and 10\% FBS (SigmaAldrich). BMSCs were incubated at $37^{\circ} \mathrm{C}$ in a $5 \%$ carbon dioxide incubator. The third generation of BMSCs was used for our experiments. When the confluence reached $70 \%$, the osteogenic medium was added to allow the osteogenic differentiation. The osteogenic medium consisted of complete medium supplemented with $0.1 \mathrm{mM}$ dexamethasone (Sigma-Aldrich), $5 \mathrm{mM}$ ß-glycerophosphate (Sigma-Aldrich), and $100 \mathrm{mM}$ ascorbic acid (Sigma-Aldrich). Then BMSCs were cultured for 14 days under the osteogenic environment, changing the medium every 3 days.

The silencing of $\beta$-catenin and BMP 2 gene was performed according to previous investigations $[44,45]$. The transfection sequences were the following: $\beta$-catenin, former primer 5 'AAGGTAGAGTGATGAAAGTTGTT-3' and reverse primer 5'- CACCATGTCCTCTGTCTATTC-3'. BMP2, former primer 5'-AGGGTTTCAGGTCAGTTTCCG-3' and reverse primer 5'-GATGATGAGGTTCTTGGCGG-3'. The cDNA of $\beta$-Catenin and BMP2 were transferred into ad lentivirus of Cre recombinase (Ad-Cre; System Biosciences, Hercules, CA, USA). BMSCs at a density of $1.0 \times 10^{5}$ cells/well were seeded into 6 -well plates for 24 hours. Next, the cells cultured in the osteogenic medium and transferred with the Ad-Cre (at a concentration of $5 \times 10^{8} \mathrm{pfu} / \mathrm{mL}$ ) for 48 hours. Ad-GFP was used as a control. Next, the cells were treated with $40 \mu \mathrm{m}$ GEN in the osteogenic medium for 7 days. Finally, the expression of Runx2, OSX, OCN, OPN, BMP2, and $\beta$-catenin were evaluated by $\mathrm{qPCR}$ and Western blotting.

\section{Cell cytotoxicity test}

BMSCs $\left(1.0 \times 10^{3}\right.$ cells/well) were seeded into 96 -well plates and cultured for 24 hours. Then, GEN at different concentrations $(0,10,20,40$, and $80 \mu \mathrm{M})$ was added, and the cells were cultured for 7 days. At the end of the 7 days, the Cell Counting Kit-8 (CCK-8; Sigma-Aldrich) was used to evaluate the cytotoxicity of GEM The absorbance was measured at $450 \mathrm{~nm}$ according to the manufacturer recommendations. A previous study described this method in detail [31].

\section{Alkaline phosphatase activity measurement}

BMSCs $\left(1.0 \times 10^{5}\right.$ per well) were seeded into 6-well plates, and then treated with GEN at different concentrations $(0,10,20$, and $40 \mu \mathrm{M})$ for 14 days under osteogenic environment. Next, BMSCs were washed with medium for three times and lysed by ultrasound. The concentration of the lysate protein was measured by the Bradford protein test (Thermo Fisher Scientific, Waltham, MA). Alkaline phosphatase (ALP) activity was detected using p-nitrophenyl-phosphate in AMP buffer (Sigma-Aldrich) at room temperature for 20 minutes. Then, sodium phosphate (0.3 M, pH 12.3, Sigma) was used to terminate the reaction. Finally, the results of ALP activity were standardized according to the protein 
concentration. Next, BMSCs were fixed with formalin for 10 minutes, ALP staining buffer (Sigma-Aldrich) was added, and the cells were incubated for 30 minutes at room temperature.

\section{Alizarin red staining}

BMSCs $\left(1.0 \times 10^{5}\right.$ per well) were seeded into 6-well plates, and treated with GEN at different concentrations $(0,10,20$, and $40 \mu \mathrm{M})$ for 14 days under osteogenic environment. Next, the medium was discarded, the cells were washed with PBS for three times, and a formalin solution was added to fix the cells. After 20 minutes, the formalin was discarded, BMSCs were washed 2 times with medium, and treated with alizarin red solution (Sigma-Aldrich) for 30 minutes. Subsequently, the stained cells were observed under an optic microscope and images were taken in random fields. Finally, the software Image $\mathrm{J}(\mathrm{NIH}$, Bethesda, MA, USA) was used to perform the statistics of the mineralized nodules, and the nodules larger than $0.04 \mathrm{~mm}$ were included in the statistical calculation [46].

\section{Quantitative PCR (q-PCR)}

BMSCs $\left(1.0 \times 10^{5}\right.$ cells/well) were seeded into 6-well plates, and treated with GEN at different concentrations $(0,10,20$, and $40 \mu \mathrm{M})$ in the osteogenic medium for 14 days. Then the mRNA expression of $A L P, O P N, O C N, O S X, R u n \times 2$, and BMP2 was detected by q-PCR. Trizol reagent (Sigma-Aldrich) was used to extract the RNA from BMSCs. The total RNA was translated into cDNA according to the reversetranscribed kit (Applied Biosystems, USA) using the following parameters: $95^{\circ} \mathrm{C}$ for 9 minutes, $36^{\circ} \mathrm{C}$ for 40 minutes for 2 cycles, then $86^{\circ} \mathrm{C}$ for 4 minutes, and final cooling to $4{ }^{\circ} \mathrm{C}$. The cDNA of the target gene was quantified by q-PCR using the SYBR Green Premix kit (Roche, Switzerland). The q-PCR parameters were the following: $95^{\circ} \mathrm{C}$ for 20 seconds, $90^{\circ} \mathrm{C}$ for 10 seconds for 40 cycles, and $60^{\circ} \mathrm{C}$ for 30 seconds. The primers used in this study (Life Technologies) were the following: $A L P$, forward primer AACCCAGACACAAGCATTCC, reverse primer GAGAGCGAAGGGTCAGTCAG. Runx2, forward primer AATTAACGCCAGTCGGAGCA, reverse primer CACTTCTCGGTCTGACGACG. OSX, forward primer CACTTCTCGGTCTGACGACG, reverse primer CACTTCTCGGTCTGACGACG. OCN, forward primer CACTTCTCGGTCTGACGACG, reverse primer ATAGCTCGTCACAAGCAGGG. BMP2, forward primer GCTTCCGTCCCTTTCATTTCT, reverse primer GCTTCCGTCCCTTTCATTTCT. OPN, forward primer GCTTCCGTCCCTTTCATTTCT, reverse primer GCTTCCGTCCCTTTCATTTCT. GAPDH, forward primer CATCACTGCCACCCAGAAGAC, reverse primer CCAGTGAGCTTCCCGTTCAG. GAPDH was used as the internal control. The relative gene expression was calculated using the $2^{-\Delta \Delta C t}$ method.

\section{Western blot}

BMSCs $\left(1.0 \times 10^{5}\right.$ cells/well) were seeded into 6-well plates, and treated with GEN at different concentrations $(0,10,20$, and $40 \mu \mathrm{M})$ in the osteogenic medium for 14 days. To assess the influence of GEN on the signalling of BMP and Wnt/ $\beta$-catenin, BMSCs were treated with either $300 \mathrm{ng} / \mathrm{mL}$ Noggin (Sigma-Aldrich) or $100 \mathrm{ng} / \mathrm{ml}$ DKK-1 (Sigma-Aldrich) in the treatment with GEN for 2 weeks. After the treatment, the total BMSC proteins were extracted by radioimmunoprecipitation assay lysis buffer (Sigma-Aldrich) at $4{ }^{\circ} \mathrm{C}$ for 30 minutes. After centrifugation, the supernatant was collected, the proteins were separated by electrophoresis and transferred to a PVDF membrane according to a previous study 
(Xiao et al. 2015). The primary antibodies used in this work were the following: anti-BMP-2 (1:2000; Sigma-Aldrich), anti- $\beta$-catenin (1:1500; Cell Signaling Technology), anti-phospho-Smad1/5/8 (1:5000; Sigma-Aldrich) anti- $\beta$-actin (1:3000; Cell Signaling Technology), anti-Runx2 (1:800; Cell Signaling Technology), and anti-OCN (1:800; Cell Signaling Technology). Next, the PVDF membrane was washed by TBST thrice, each time for 5 minutes and the second antibody goat anti-rabbit (1:3000, Abcam) was added. The specific protein bands were visualized using a proprietary chemiluminescence kit (PerkinElmer, Inc, Waltham, MA). The bands were quantified by densitometry using the Image-Pro Plus 6.0 software (Media Cybernetics, Rockville, MD).

\section{Experimental model and animal groups}

Thirty-six female C57BL/ 6 mice (8-week-old, $21 \pm 2 \mathrm{~g}$ ) were obtained from the animal experimental centre of the Southern Medical University (Guangzhou, Guangdong, China). The experimental mice were stochastically divided into three groups: Sham group $(n=12)$, ovariectomized $(O V X)$ group $(n=12)$ and OVX + GEN group $(n=12)$. The OVX + GEN groups were treated by an oral gavage of $50 \mathrm{mg} / \mathrm{kg} / \mathrm{day}$ GEN during these 3 months. The Sham group and OVX group received the same dose of saline by oral gavage. After 3 months, the experimental mice were euthanized by cervical dislocation and the femurs were collected for further studies.

\section{Histological and immunohistochemical staining}

The femur was immersed in $4 \%$ paraformaldehyde for 48 hours and decalcified using $15 \%$ ethylenediaminetetraacetic acid for 14 days. Then, they were dehydrated, paraffin embedded, and cut into $4 \mu \mathrm{m}$-thick sections. To perform the haematoxylin-eosin (HE) staining, the sections were dewaxed, hydrated, and stained with HE dyes (Abcam). Finally, the HE stained sections were photographed and analysed. As regard the immunohistochemistry, the sections were dewaxed, hydrated, treated with $3 \%$ hydrogen peroxide for 15 minutes and with protease $\mathrm{K}$ for 10 minutes. Next, the sections were treated with primary antibodies and incubated overnight at $4{ }^{\circ} \mathrm{C}$. The primary antibodies (Santa Cruz Biotechnology) used were the following: anti-Runx2 (1:200), anti-BMP-2 (1:200), and anti-OCN (1:200).

The sections were washed with PBS thrice for a total of 15 minutes and the second antibody was added and incubated for 50 minutes. Next, the sections were washed three times with PBS, and then diaminobenzidine solution was added to obtain the chromogenic reaction. Finally, the sections were observed and analysed under an optical microscope.

\section{Microcomputer tomography analysis}

The collected femurs were preserved in $4 \%$ paraformaldehyde for 48 hours. The prepared femur was scanned and analysed by high-resolution micro CT (Caskaisheng, China). The scanning parameters of the micro-CT were set as follows: $80 \mathrm{kV}, 15 \mu \mathrm{A}$, and a scanning thickness of $20 \mu \mathrm{m}$. The area below the crud end of femoral shaft was chosen as the analysis area for statistical analysis [47]. The bone parameters for statistical analysis included the following three indexes: trabecular bone mineral density (BMD), trabecular number, and trabecular thickness.

\section{Statistical analysis}


Statistical analysis was performed using GraphPad Prism 6 (Manufacturer, La Jolla, CA, USA). All in vitro experiments were repeated three times, and each experiment was carried out in triplicate. In the in vivo experiments, each group contained at least 6 rats. Results were expressed as mean \pm standard deviation (SD). One-way ANOVA and Dunnett's test were used to compare multiple groups, while unpaired Student's $t$-test was used for the comparison of two groups. $P<0.05$ was considered statistically significant.

\section{Abbreviations}

GEN: Gentiopicroside; BMSCs: bone mesenchymal stem cells; OCN: osteocalcin; OSX: osterix; OPN; osteopontin; Runx2: runt-related transcription factor 2; ALP: Alkaline phosphatase; OVX: ovariectomized; HE: haematoxylin-eosin; BMD: bone mineral density; SD: standard deviation.

\section{Declarations}

\section{Availability of data and materials}

Please contact the corresponding author for data on reasonable request.

\section{Ethics approval and consent to participate}

This project was carried out with the permission of the Ethics Committee of the Ningbo No. 6 Hospital (registered number 2015-018).

\section{Consent for publication}

Not applicable.

\section{Competing interests}

The authors declare that they have no conflicts of interest.

\section{Funding}

This work was funded by Australia National Health and Medical Research Council (NHMRC) (1158402), National Natural Science Foundation of China (NSFC) (81671928), Ningbo Science \&Technology and People-Benefit Program (2017C50048), and Ningbo Medical Science \&Technology Program (2016A22).

\section{Authors' contributions}


HJ, QW and LW conceived and designed the study. HJ, JZ and WL performed the experiments. JZ, WL, JD, YKS, LY collected and analysed data. HJ, JD, CJX, QW and LW drafted and revised of the manuscript. All authors read and approved the final manuscript.

\section{Acknowledgements}

Not applicable.

\section{Supplementary Materials}

\section{Supplementary Fig. 1. GEN had no effect on expression of ALP, RUNX2, OCN, and BMP2 without the} osteogenic medium. BMSCs were cultivated with $\operatorname{GEN}(0,10,20$, and $40 \mu \mathrm{M})$ without the condition of osteogenic induction for 2 weeks. $(a, b)$ The result of ALP staining and ALP activity. The protein level of Runx2 (c, d), OCN (c, e), and BMP2 (c, f) were measured by Western blot.

Supplementary Fig. 2. Ovariectomized osteoporosis mice model was successfully constructed. (a) Body weight and (b) uterine weight were shown in this data. ${ }^{*} P<0.05$ versus Sham group.

Supplementary Fig. 3. GEN had no effect on $\beta$-catenin-BMP2 signaling without the osteogenic medium. BMSCs were cultivated with $\operatorname{GEN}(0,10,20$, and $40 \mu \mathrm{M})$ without the condition of osteogenic induction for 2 weeks. The level of $p$-Smad1/5/8 $(a, b), \beta$-catenin protein $(a, c)$ were evaluated by Western blot.

\section{References}

1. Consensus A. (1993) Consensus development conference: diagnosis, prophylaxis, and treatment of osteoporosis. Am J Med 94 (6):646-650.

2. Hernlund E, Svedbom A, Ivergård M, Compston J, Cooper C, Stenmark J, McCloskey EV, Jönsson B, Kanis JA. Osteoporosis in the European Union: medical management, epidemiology and economic burden. Archives of osteoporosis. 2013;8(1-2):136.

3. El'chaninov D, Akker L, Fedorova I, Popovtseva A. (2009) Bone resorption and formation markers in women with climacteric syndrome in early postmenopause. Klinicheskaia laboratornaia diagnostika (10):21-24.

4. Rachner TD, Khosla S, Hofbauer LC. Osteoporosis: now and the future. The Lancet. 2011;377(9773):1276-87.

5. Dören M, Samsioe G. Prevention of postmenopausal osteoporosis with oestrogen replacement therapy and associated compounds: update on clinical trials since 1995. Human Reproduction Update. 2000;6(5):419-26.

6. Han Y, Jin Y, Lee SH, Khadka DB, Cho W-J, Lee KY. Berberine bioisostere Q8 compound stimulates osteoblast differentiation and function in vitro. Pharmacological research. 2017;119:463-75. 
7. Li X-F, Xu H, Zhao Y-J, Tang D-Z, Xu G-H, Holz J, Wang J, Cheng S-D, Shi Q, Wang Y-J. (2013) Icariin augments bone formation and reverses the phenotypes of osteoprotegerin-deficient mice through the activation of Wnt/ $\beta$-catenin-BMP signaling. Evidence-Based Complementary and Alternative Medicine 2013.

8. Raut N, Wicks SM, Lawal TO, Mahady GB. (2019) Epigenetic regulation of bone remodeling by natural compounds. Pharmacological research:104350.

9. Miyamoto K, Yoshida S, Kawasumi M, Hashimoto K, Kimura T, Sato Y, Kobayashi T, Miyauchi Y, Hoshi H, Iwasaki R. Osteoclasts are dispensable for hematopoietic stem cell maintenance and mobilization. J Exp Med. 2011;208(11):2175-81.

10. Adler BJ, Kaushansky K, Rubin CT. Obesity-driven disruption of haematopoiesis and the bone marrow niche. Nature Reviews Endocrinology. 2014;10(12):737.

11. Lo Y-C, Chang Y-H, Wei B-L, Huang Y-L, Chiou W-F. Betulinic acid stimulates the differentiation and mineralization of osteoblastic MC3T3-E1 cells: involvement of BMP/Runx2 and $\beta$-catenin signals. J Agric Food Chem. 2010;58(11):6643-9.

12. Grassi F, Tyagi AM, Calvert JW, Gambari L, Walker LD, Yu M, Robinson J, Li JY, Lisignoli G, Vaccaro C. Hydrogen sulfide is a novel regulator of bone formation implicated in the bone loss induced by estrogen deficiency. J Bone Miner Res. 2016;31(5):949-63.

13. Huang L, Wang X, Cao H, Li L, Chow DH-K, Tian L, Wu H, Zhang J, Wang N, Zheng L. A bone-targeting delivery system carrying osteogenic phytomolecule icaritin prevents osteoporosis in mice. Biomaterials. 2018;182:58-71.

14. Caplan Al, Bruder SP. Mesenchymal stem cells: building blocks for molecular medicine in the $21 \mathrm{st}$ century. Trends Mol Med. 2001;7(6):259-64.

15. Rosset P, Deschaseaux F, Layrolle P. Cell therapy for bone repair. Orthopaedics Traumatology: Surgery Research. 2014;100(1):107-12.

16. Huang W, Zheng X, Yang X, Fan S. Stimulation of Osteogenic Differentiation by Saikosaponin-A in

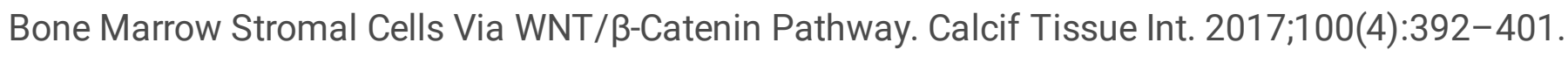

17. Ma Z-p, Liao J-c, Zhao C, Cai D-z. Effects of the 1, 4-dihydropyridine L-type calcium channel blocker benidipine on bone marrow stromal cells. Cell tissue research. 2015;361(2):467-76.

18. Raisz LG. Pathogenesis of osteoporosis: concepts, conflicts, and prospects. J Clin Investig. 2005;115(12):3318-25.

19. Khosla S, Westendorf JJ, Oursler MJ. Building bone to reverse osteoporosis and repair fractures. J Clin Investig. 2008;118(2):421-8.

20. Mao J, Wang J, Liu B, Pan W, Farr GH III, Flynn C, Yuan H, Takada S, Kimelman D, Li L. Low-density lipoprotein receptor-related protein-5 binds to Axin and regulates the canonical Wnt signaling pathway. Molecular cell. 2001;7(4):801-9.

21. Clevers H, Nusse R. Wnt/ $\beta$-catenin signaling and disease. Cell. 2012;149(6):1192-205. 
22. Casanovas G, Mleczko-Sanecka K, Altamura S, Hentze MW, Muckenthaler MU. Bone morphogenetic protein (BMP)-responsive elements located in the proximal and distal hepcidin promoter are critical for its response to HJV/BMP/SMAD. Journal of molecular medicine. 2009;87(5):471-80.

23. Lee M-H, Kim Y-J, Kim H-J, Park H-D, Kang A-R, Kyung H-M, Sung J-H, Wozney JM, Ryoo H-M. BMP-2induced Runx2 expression is mediated by DIx5, and TGF- $\beta 1$ opposes the BMP-2-induced osteoblast differentiation by suppression of Dlx5 expression. J Biol Chem. 2003;278(36):34387-94.

24. Phimphilai M, Zhao Z, Boules H, Roca H, Franceschi RT. BMP signaling is required for RUNX2dependent induction of the osteoblast phenotype. J Bone Miner Res. 2006;21(4):637-46.

25. Weckesser S, Engel K, Simon-Haarhaus B, Wittmer A, Pelz K, Schempp C. Screening of plant extracts for antimicrobial activity against bacteria and yeasts with dermatological relevance. Phytomedicine. 2007;14(7-8):508-16.

26. Liu N, Li Y-X, Gong S-S, Du J, Liu G, Jin S-J, Zhao C-J, Niu Y, Sun T, Yu J-Q. Antinociceptive effects of gentiopicroside on neuropathic pain induced by chronic constriction injury in mice: a behavioral and electrophysiological study. Can J Physiol Pharmacol. 2016;94(7):769-78.

27. Yang Y, Wang Z, Zhang L, Yin B, Lv L, He J, Chen Z, Wen X, Qiao B, Sun W. Protective effect of gentiopicroside from Gentiana macrophylla Pall. in ethanol-induced gastric mucosal injury in mice. Phytother Res. 2018;32(2):259-66.

28. Du L, Nong M-N, Zhao J-M, Peng X-M, Zong S-H, Zeng G-F. Polygonatum sibiricum polysaccharide inhibits osteoporosis by promoting osteoblast formation and blocking osteoclastogenesis through Wnt/ $\beta$-catenin signalling pathway. Scientific reports. 2016;6(1):1-12.

29. Tao X, Qi Y, Xu L, Yin L, Han X, Xu Y, Wang C, Sun H, Peng J. Dioscin reduces ovariectomy-induced bone loss by enhancing osteoblastogenesis and inhibiting osteoclastogenesis. Pharmacological research. 2016;108:90-101.

30. Chen F, Xie L, Kang R, Deng R, Xi Z, Sun D, Zhu J, Wang L. Gentiopicroside inhibits RANKL-induced osteoclastogenesis by regulating NF-KB and JNK signaling pathways. Biomed Pharmacother. 2018;100:142-6.

31. Xiao J-j, Zhao W-j, Zhang X-t, Zhao W-I, Wang X-x, Yin S-h, Jiang F, Zhao Y-x, Chen F-n, Li S-I. Bergapten promotes bone marrow stromal cell differentiation into osteoblasts in vitro and in vivo. Molecular cellular biochemistry. 2015;409(1-2):113-22.

32. Zhu B, Xue F, Zhang C, Li G. LMCD1 promotes osteogenic differentiation of human bone marrow stem cells by regulating BMP signaling. Cell death disease. 2019;10(9):1-11.

33. Qi Q, Wang Y, Wang X, Yang J, Xie Y, Zhou J, Li X, Wang B. (2019) Histone demethylase KDM4A regulates adipogenic and osteogenic differentiation via epigenetic regulation of $\mathrm{C} / \mathrm{EBPa}$ and canonical Wnt signaling. Cellular and Molecular Life Sciences:1-15.

34. Joeng KS, Lee Y-C, Lim J, Chen Y, Jiang M-M, Munivez E, Ambrose C, Lee BH. Osteocyte-specific WNT1 regulates osteoblast function during bone homeostasis. J Clin Investig. 2017;127(7):267888. 
35. Su X, Liao L, Shuai Y, Jing H, Liu S, Zhou H, Liu Y, Jin Y. MiR-26a functions oppositely in osteogenic differentiation of BMSCs and ADSCs depending on distinct activation and roles of Wnt and BMP signaling pathway. Cell death disease. 2015;6(8):e1851-1.

36. He Y, Li Y, Chen G, Wei C, Zhang X, Zeng B, Yi C, Wang C, Yu D. Concentration-dependent cellular behavior and osteogenic differentiation effect induced in bone marrow mesenchymal stem cells treated with magnetic graphene oxide. Journal of Biomedical Materials Research Part A. 2020;108(1):50-60.

37. Oichi T, Taniguchi Y, Soma K, Oshima Y, Yano F, Mori Y, Chijimatsu R, Kim-Kaneyama J-r, Tanaka S, Saito T. Adamts 17 is involved in skeletogenesis through modulation of BMP-Smad1/5/8 pathway. Cell Mol Life Sci. 2019;76(23):4795-809.

38. Krishnan V, Bryant HU, MacDougald OA. Regulation of bone mass by Wnt signaling. J Clin Investig. 2006;116(5):1202-9.

39. Rauner M, Rachner TD, Hofbauer LC. Bone Formation and the Wnt Signaling Pathway. N Engl J Med. 2016;375(19):1902. doi:10.1056/NEJMc1609768.

40. Rulifson IC, Karnik SK, Heiser PW, Ten Berge D, Chen H, Gu X, Taketo MM, Nusse R, Hebrok M, Kim SK. (2007) Wnt signaling regulates pancreatic $\beta$ cell proliferation. Proceedings of the National Academy of Sciences 104 (15):6247-6252.

41. Lim DA, Tramontin AD, Trevejo JM, Herrera DG, García-Verdugo JM, Alvarez-Buylla A. Noggin antagonizes BMP signaling to create a niche for adult neurogenesis. Neuron. 2000;28(3):713-26.

42. Tian E, Zhan F, Walker R, Rasmussen E, Ma Y, Barlogie B, Shaughnessy JD Jr. The role of the Wntsignaling antagonist DKK1 in the development of osteolytic lesions in multiple myeloma. $\mathrm{N} \mathrm{Engl} \mathrm{J}$ Med. 2003;349(26):2483-94.

43. Zhao S-J, Kong F-Q, Jie J, Li Q, Liu H, Xu A-D, Yang Y-Q, Jiang B, Wang D-D, Zhou Z-Q. Macrophage MSR1 promotes BMSC osteogenic differentiation and M2-like polarization by activating $\mathrm{PI3K} / \mathrm{AKT} / \mathrm{GSK} 3 \beta / \beta$-catenin pathway. Theranostics. 2020;10(1):17.

44. Yan $Y$, Tang D, Chen M, Huang J, Xie R, Jonason JH, Tan X, Hou W, Reynolds D, Hsu W. Axin2 controls bone remodeling through the $\beta$-catenin-BMP signaling pathway in adult mice. Journal of cell science. 2009;122(19):3566-78.

45. Tang DZ, Hou W, Zhou Q, Zhang M, Holz J, Sheu TJ, Li TF, Cheng SD, Shi Q, Harris SE. Osthole stimulates osteoblast differentiation and bone formation by activation of $\beta$-catenin-BMP signaling. J Bone Miner Res. 2010;25(6):1234-45.

46. Pang J, Wu B, He W, Zhang Y, Zhao H, Xie Z. Effect of antisense oligonucleotide against mouse dentine matrix protein 1 on mineralization ability and calcium ions metabolism in odontoblast-like cell line MDPC-23. International endodontic journal. 2006;39(7):527-37.

47. Li S-f, Tang J-J, Chen J, Zhang P, Wang T, Chen T-y, Yan B, Huang B, Wang L, Huang M-j (2015) Regulation of bone formation by baicalein via the mTORC1 pathway. Drug design, development and therapy 9:5169. 
a<smiles>C=CC1C2=CCOC(=O)C2=COC1OC1OC(CO)[C@@H](O)C(O)C1O</smiles>

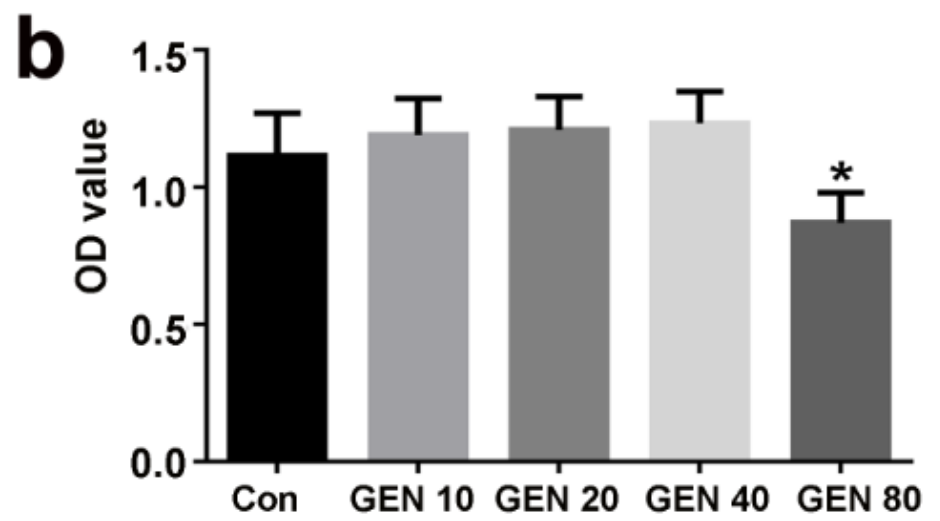

C
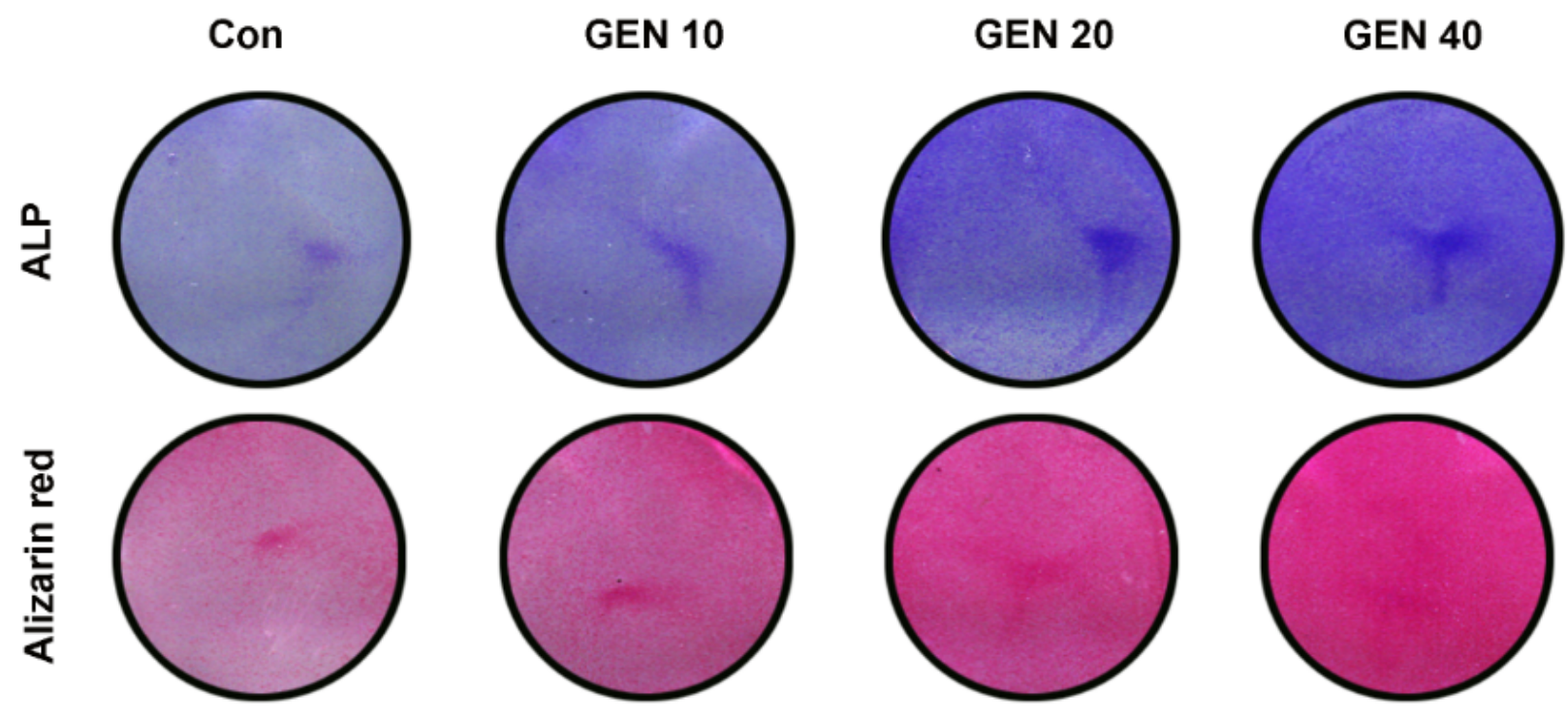

e

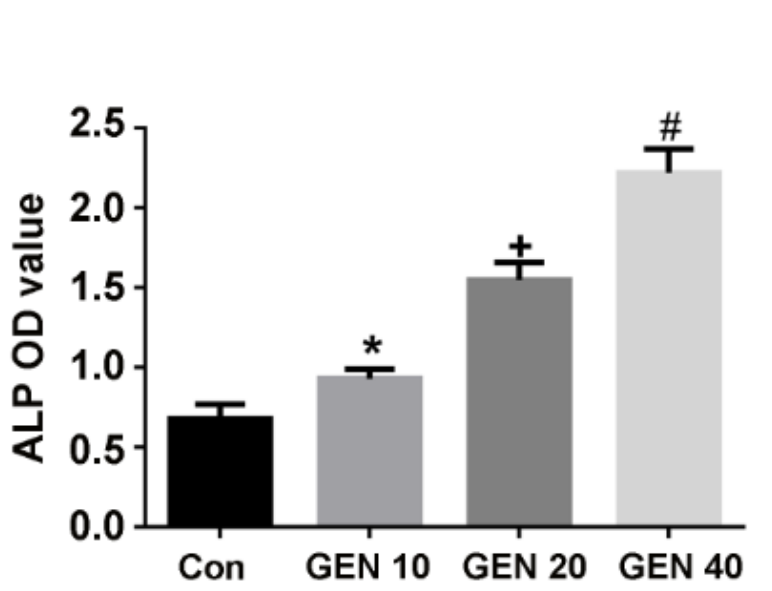

f

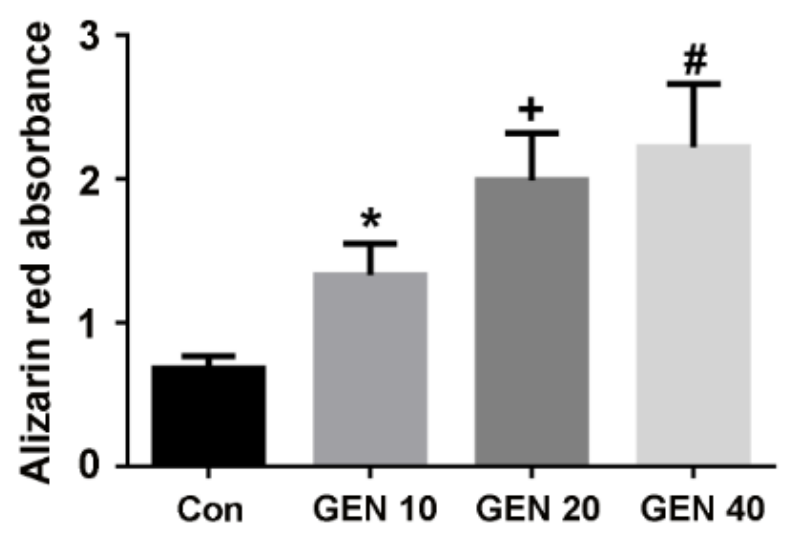

Figure 1

Effect of GEN on the proliferation and differentiation of BMSCs. a, Chemical structure of GEN. b, BMSCs were cultivated with increasing doses of $\operatorname{GEN}(0,10,20,40$, and $80 \mu \mathrm{M})$ for 1 week. The proliferation of BMSCs was evaluated by CCK-8 test. BMSCs were cultivated with $\operatorname{GEN}(0,10,20$, and $40 \mu \mathrm{M})$ in the 
condition of osteogenic induction for 2 weeks. The activity of ALP was tested by ALP staining (c,e), while mineralized nodules were detected by Alizarin Red staining (d, $f)$. ${ }^{*}<0.05$ versus group without $G E N,+P$ $<0.05$ versus $10 \mu \mathrm{M}$ GEN, \#P $<0.05$ versus $20 \mu \mathrm{M}$ GEN.

a

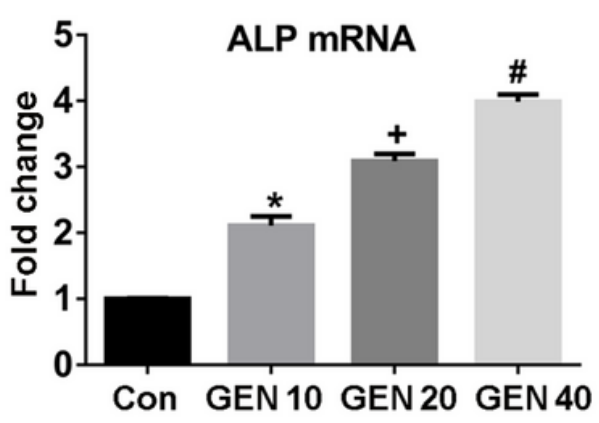

d

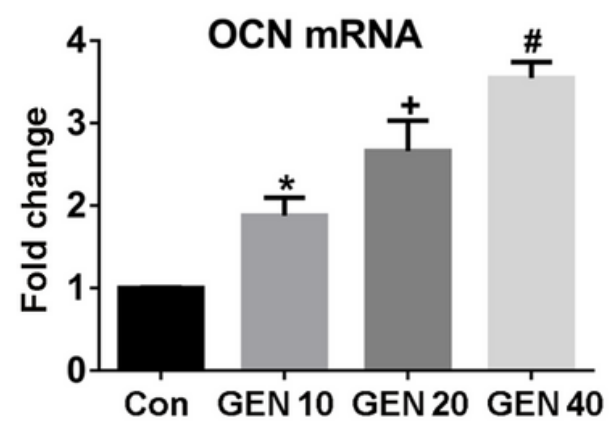

b

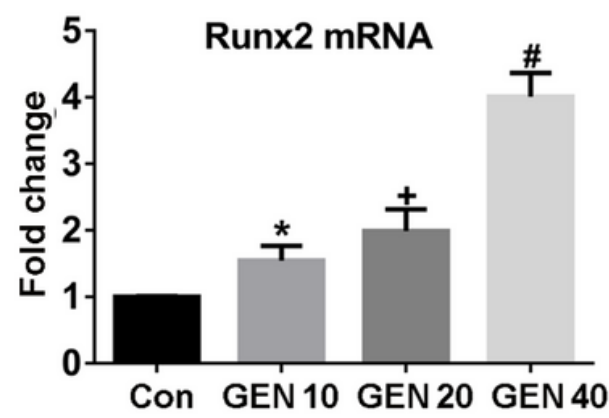

$\mathbf{e}$

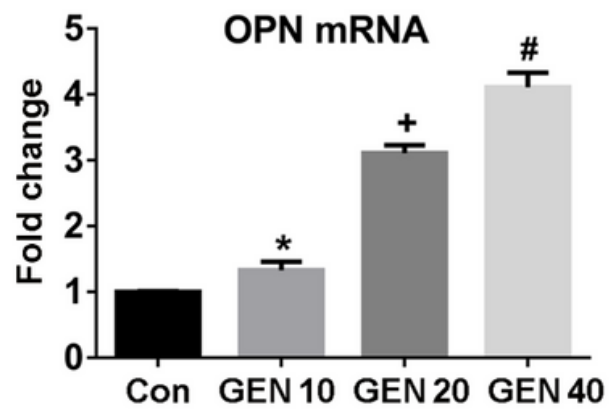

C

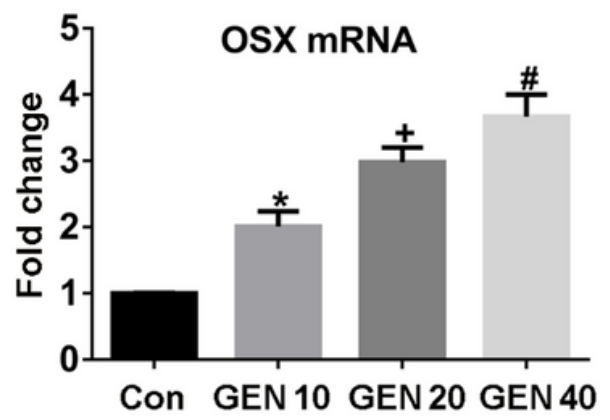

f

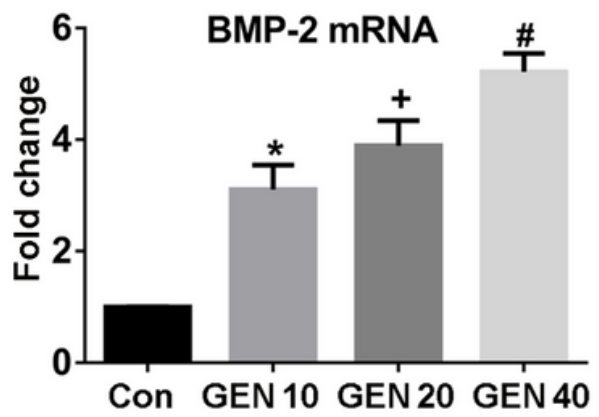

Figure 2

GEN increased the production of ossific related genes in vitro. BMSCs were cultivated with $\operatorname{GEN}(0,10,20$, and $40 \mu \mathrm{M}$ ) in the condition of osteogenic induction for 2 weeks. (a-f) mRNA levels of ALP, Runx2, OSX】 OCN, OPN, and BMP2 were detected by q-PCR analysis. ${ }^{*} P<0.05$ versus control group, $+P<0.05$ versus $10 \mu \mathrm{M}$ GEN, \#P $<0.05$ versus $20 \mu \mathrm{M}$ GEN. 


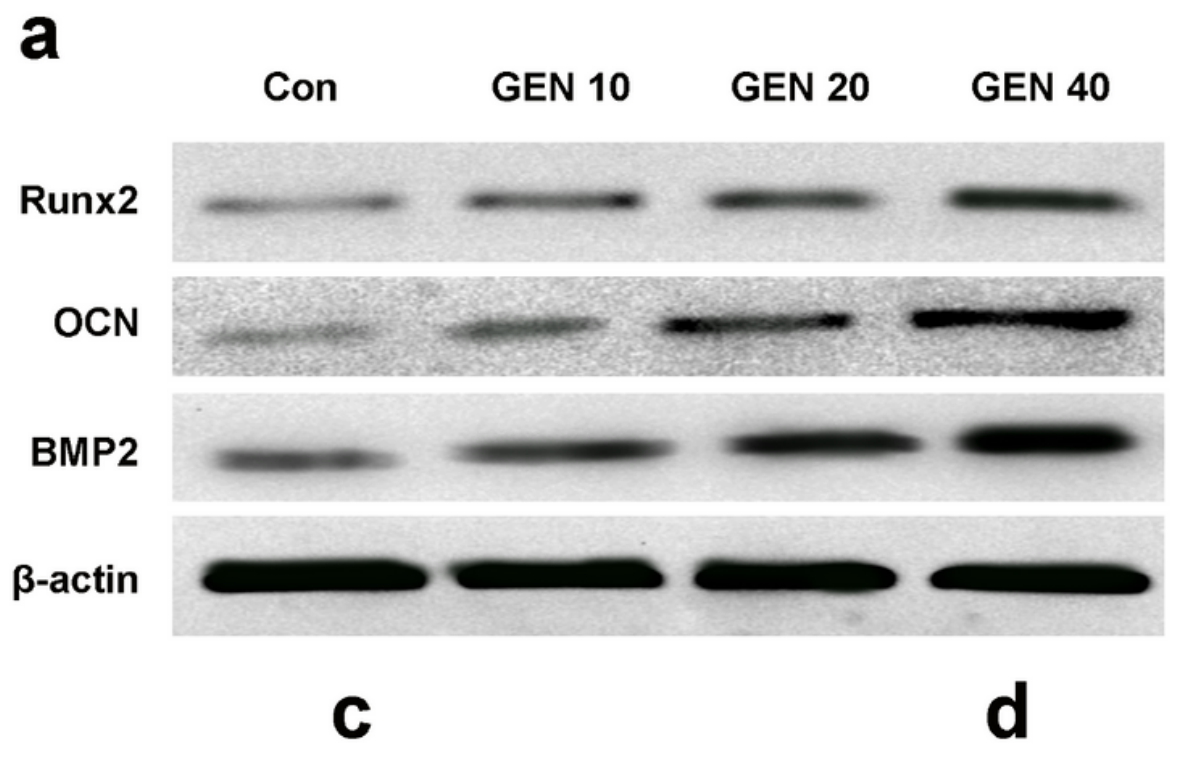

b
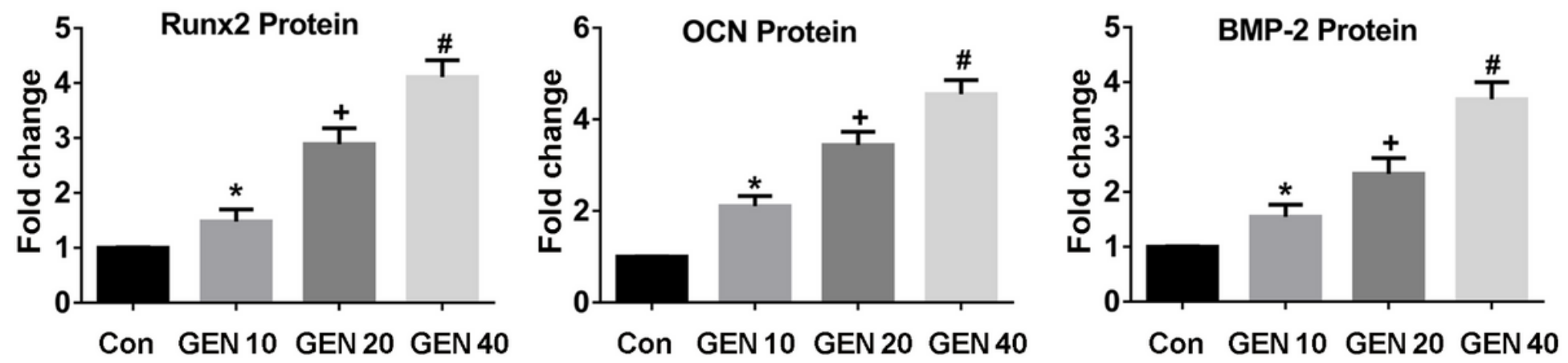

Figure 3

GEN increased the production of osteogenic related proteins in vitro. BMSCs were cultivated with GEN ( 0 , $10,20$, and $40 \mu \mathrm{M})$ in the condition of osteogenic induction for 2 weeks. Protein level of Runx2 $(a, b), O C N$ $(\mathrm{a}, \mathrm{c})$, and BMP2 $(\mathrm{a}, \mathrm{d})$ were detected by Western blot. ${ }^{*} \mathrm{P}<0.05$ versus group without $\mathrm{GEN},+\mathrm{P}<0.05$ versus $10 \mu \mathrm{M}$ GEN, $\# \mathrm{P}<0.05$ versus $20 \mu \mathrm{M}$ GEN. 
a

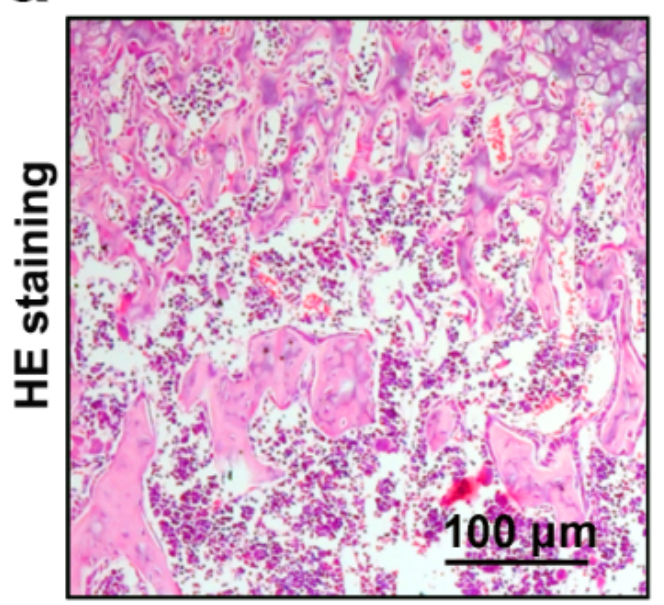

b

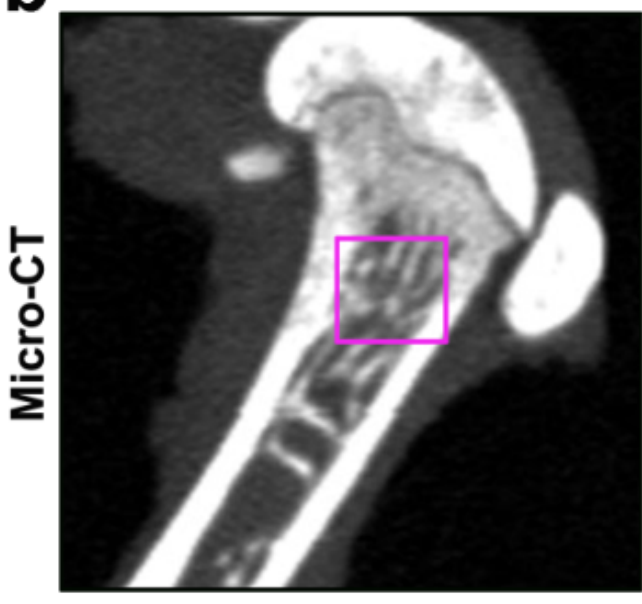

C

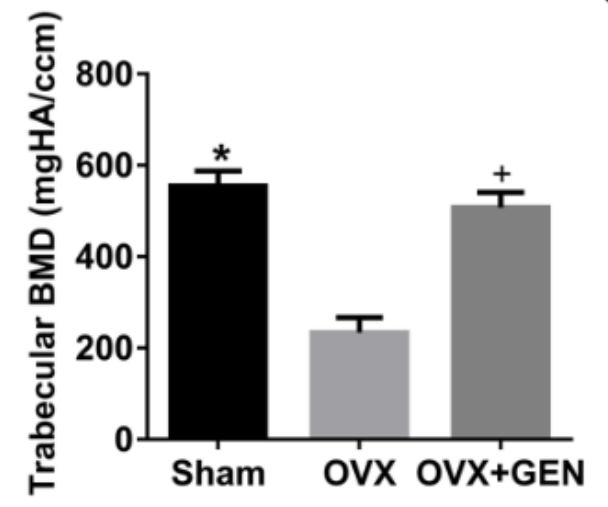

OVX
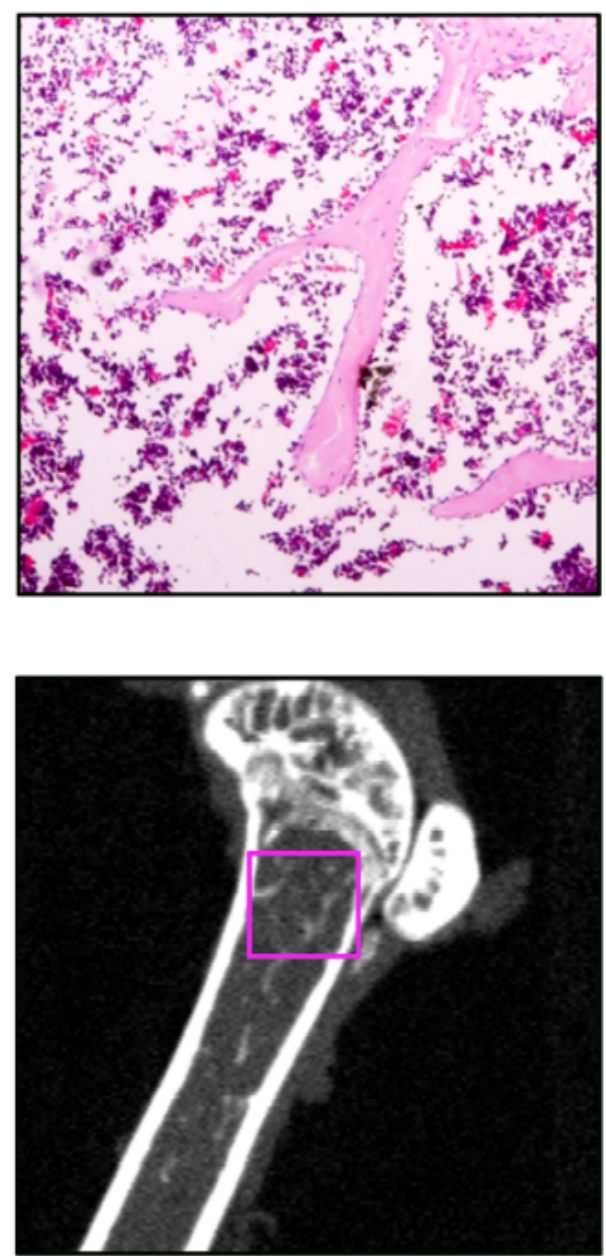

d

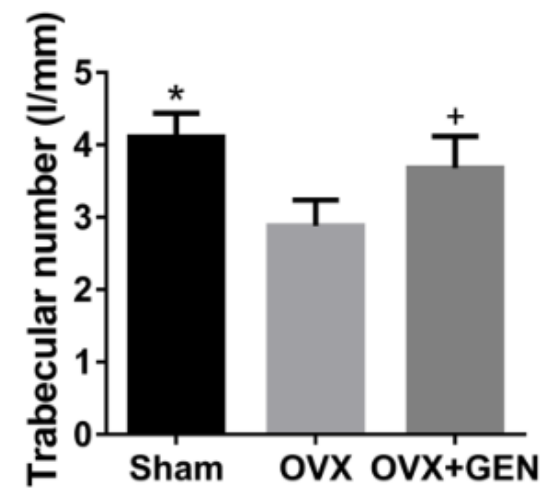

OVX+GEN
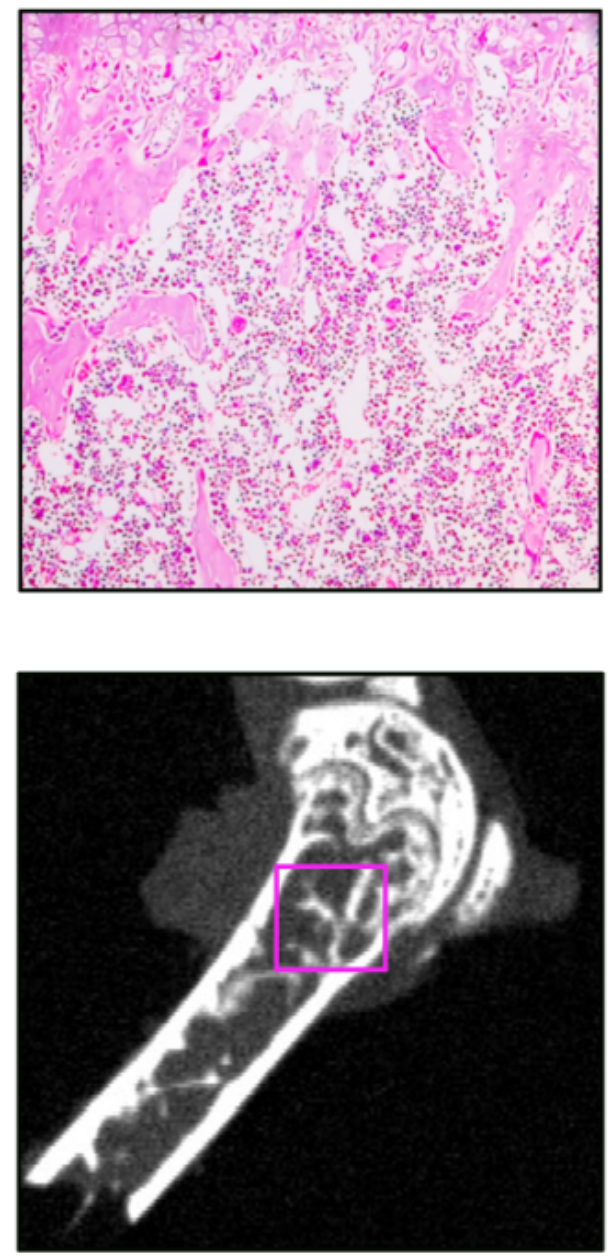

e

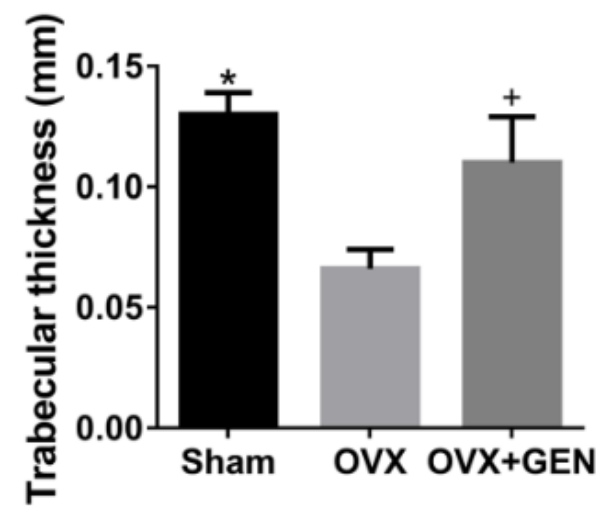

Figure 4

GEN promotes bone formation in OVX mice. (a) After 3 months of GEN treatment, the sections of mice femurs were evaluated by HE staining. (b) Micro-CT photos of the statistical analysis site of femur. (c-e) Statistical credits of bone mineral density (BMD), trabeculae number, and trabeculae thickness in different groups. ${ }^{*} \mathrm{P}<0.05$ and $+\mathrm{P} \otimes 0.05$ versus with $\mathrm{OVX}$ group. 

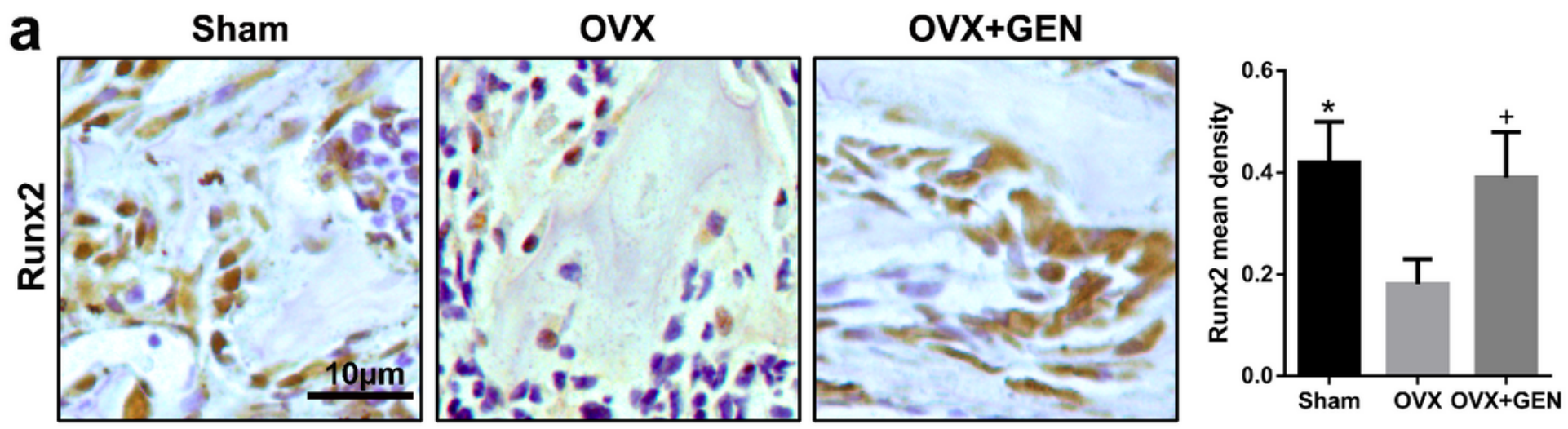

b
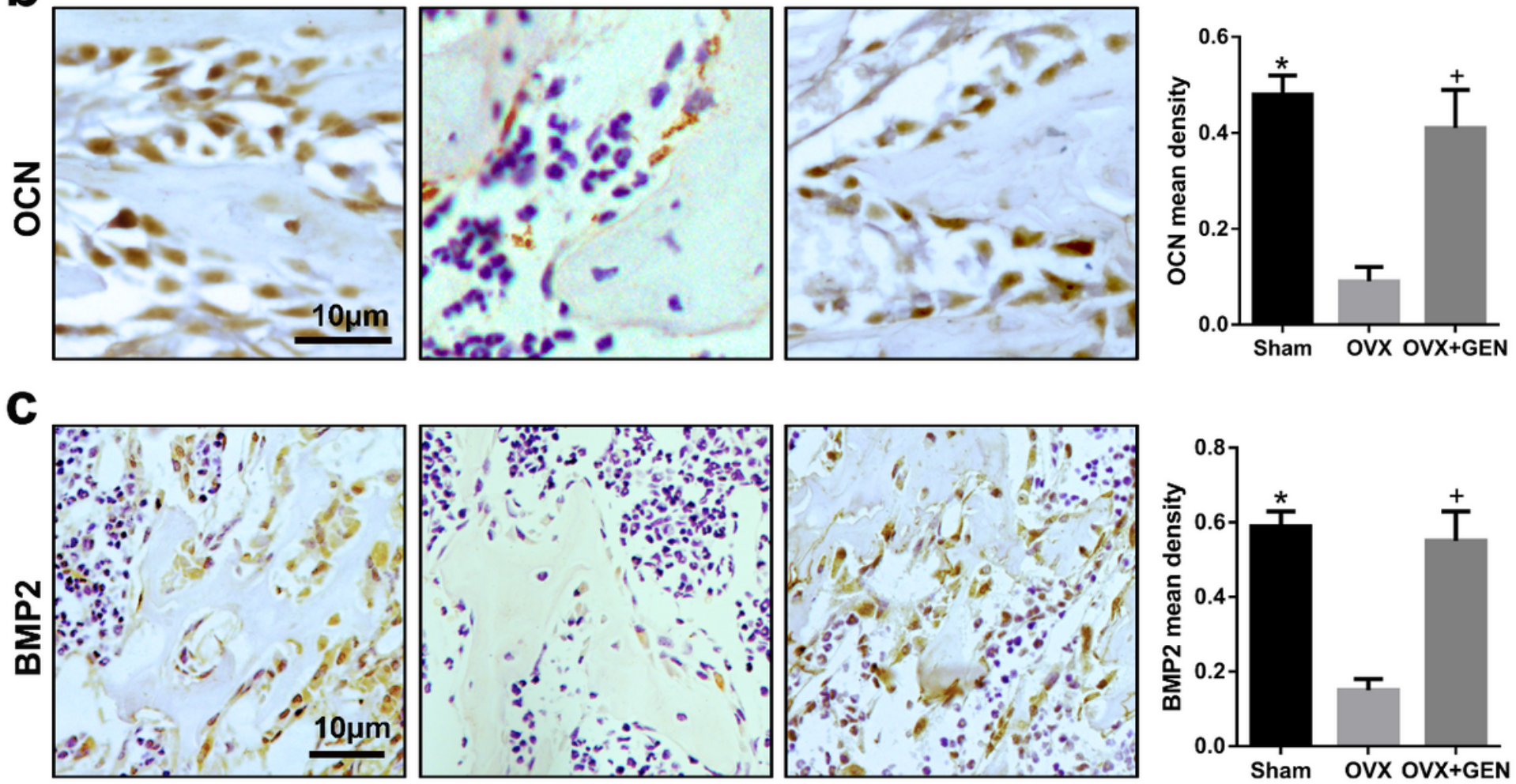

Figure 5

GEN stimulates the expression of osteogenic markers in vivo. 3 months after administration of GEN in OVX mice冈the level of Runx2 (a, d), OCN (b, e), and BMP2 (c, f) were tested by immunohistochemistry. $* P<0.05$ and $+P \otimes 0.05$ versus with $O V X$ group. 

a
Con
GEN 10
GEN 20
GEN 40

p-Smad1/5/8

$\beta$-catenin

$\beta$-actin
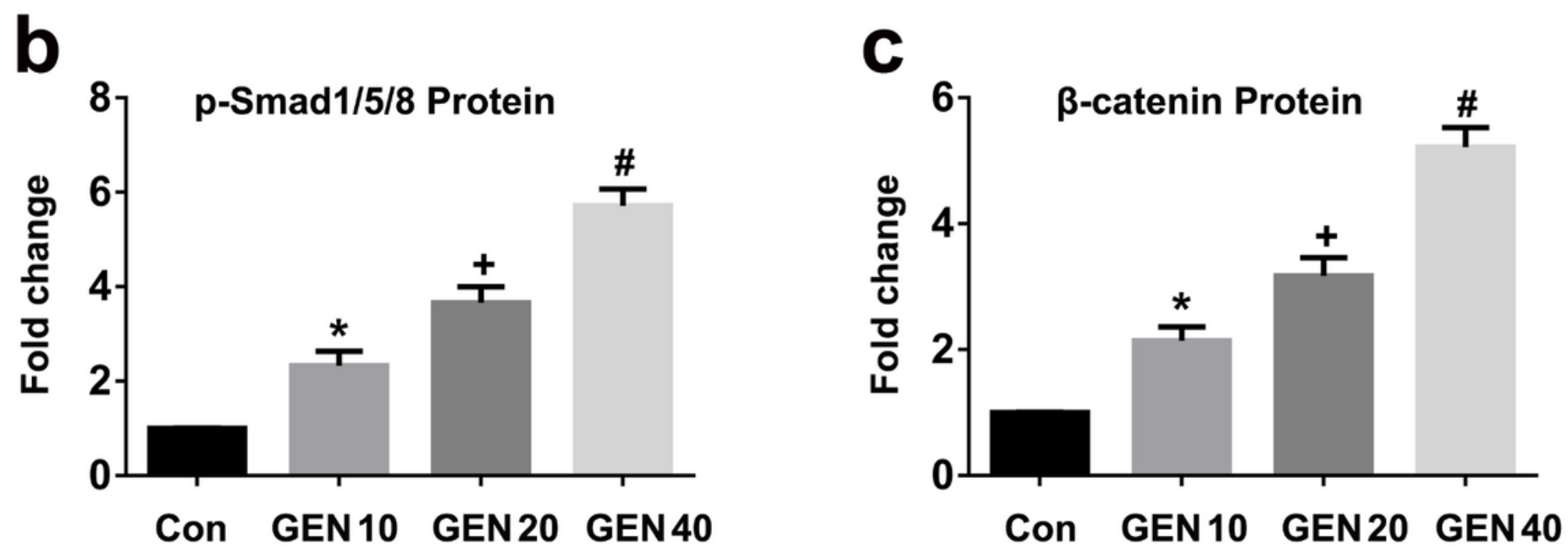

Figure 6

Effect of GEN on BMP signaling and WNT/ $\beta$-catenin signaling pathway. BMSCs were cultivated with GEN $(0,10,20$, and $40 \mu \mathrm{M})$ in the condition of osteogenic induction for 2 weeks. The protein level of $p$ Smad1/5/8 $(a, b)$ and $\beta$-catenin $(a, c)$ was measured by Western blot. * $P<0.05$ versus group without $G E N$, $+\mathrm{P}<0.05$ compared with $10 \mu \mathrm{M}$ GEN, \#P $<0.05$ compared with $20 \mu \mathrm{M}$ GEN. 
a
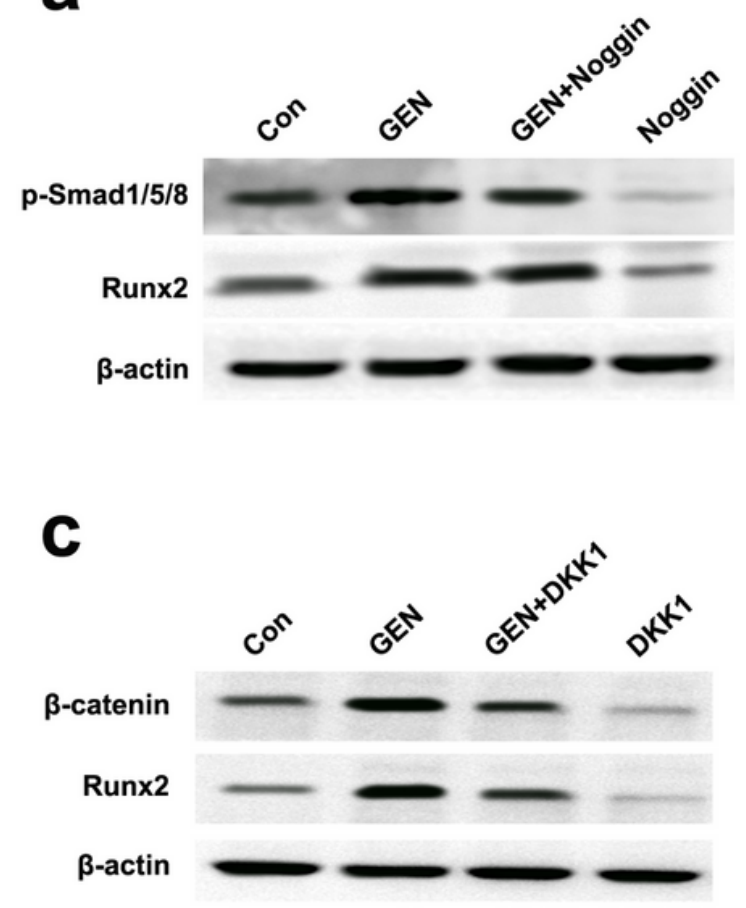
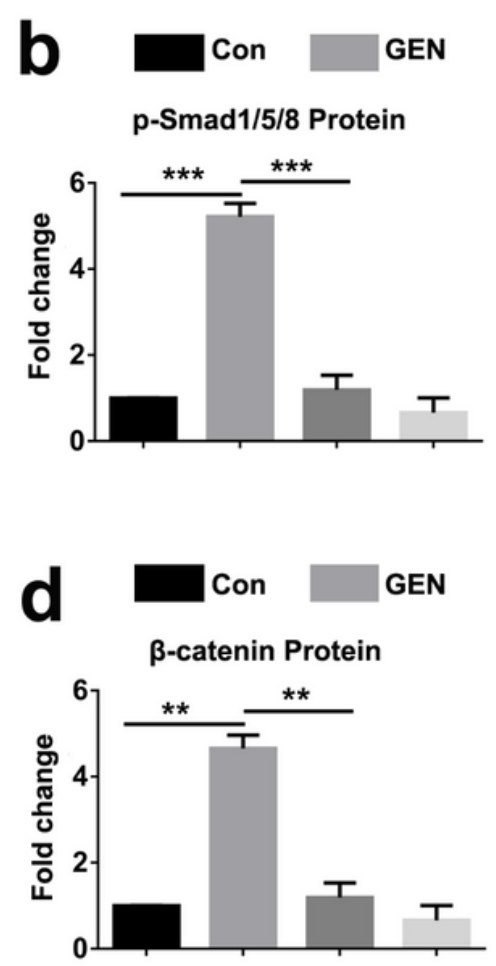

GEN+Noggin

Noggin

Runx2 Protein

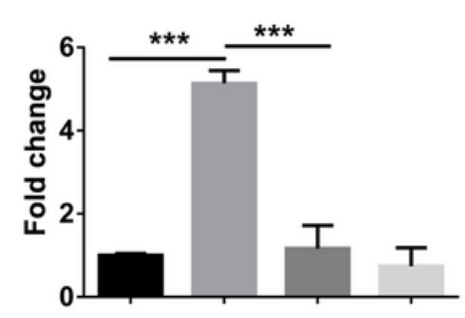

GEN+DKK1

DKK1

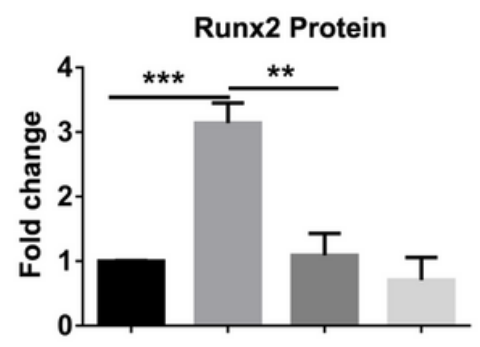

Figure 7

Inhibition of BMP signaling and WNT/ $\beta$-catenin signaling pathway blocked GEN-induced osteogenic differentiation in BMSCs. BMSCs were treated with GEN $(40 \mu \mathrm{M})$ under osteogenic condition in the presence of Noggin or DKK1 for 2 weeks. The level of p-Smad1/5/8 (a,b), $\beta$-catenin (d, e), and Runx2 (c, f) were evaluated by Western blot. $* * P<0.01, * \star \star P<0.001$. 
a
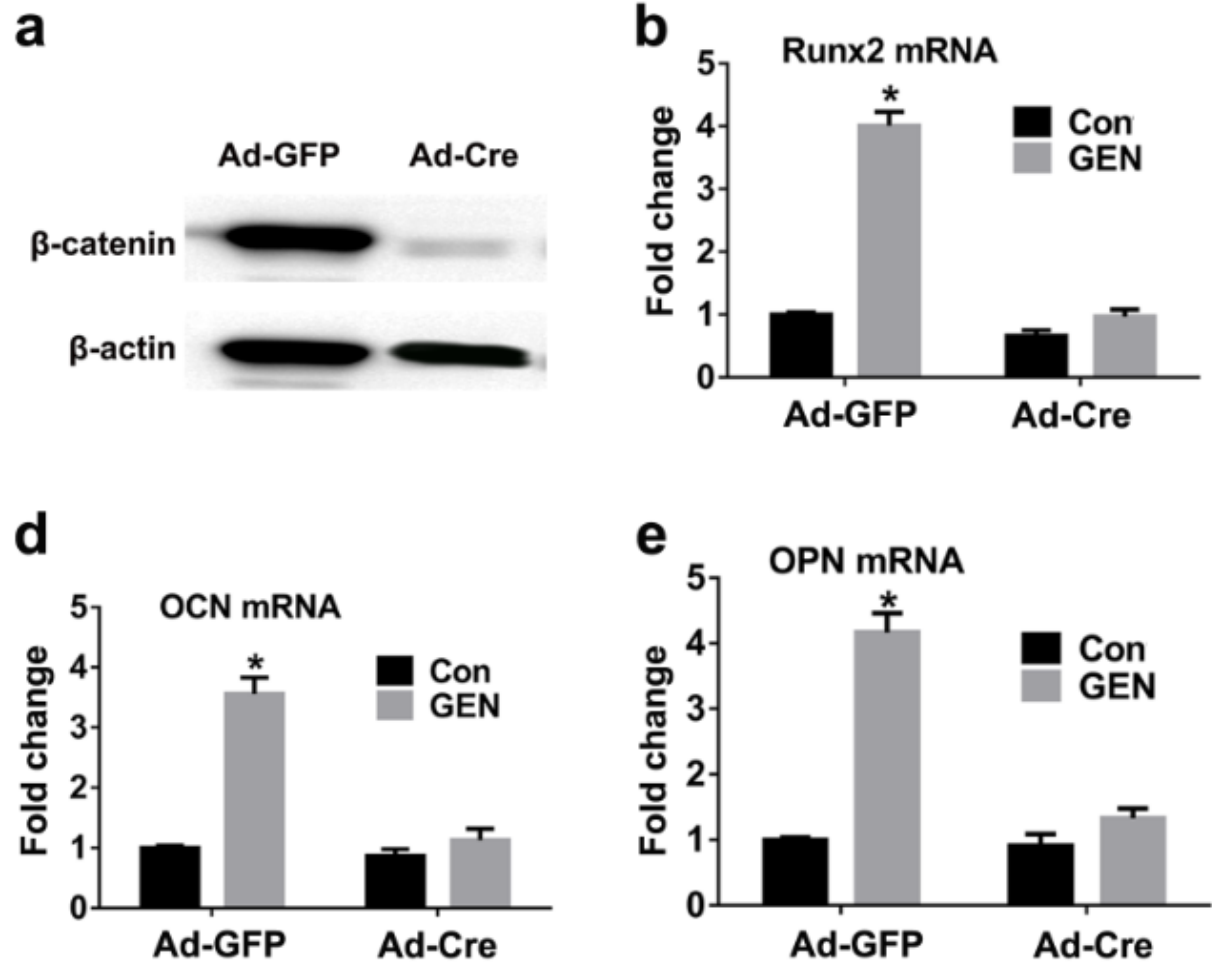

g

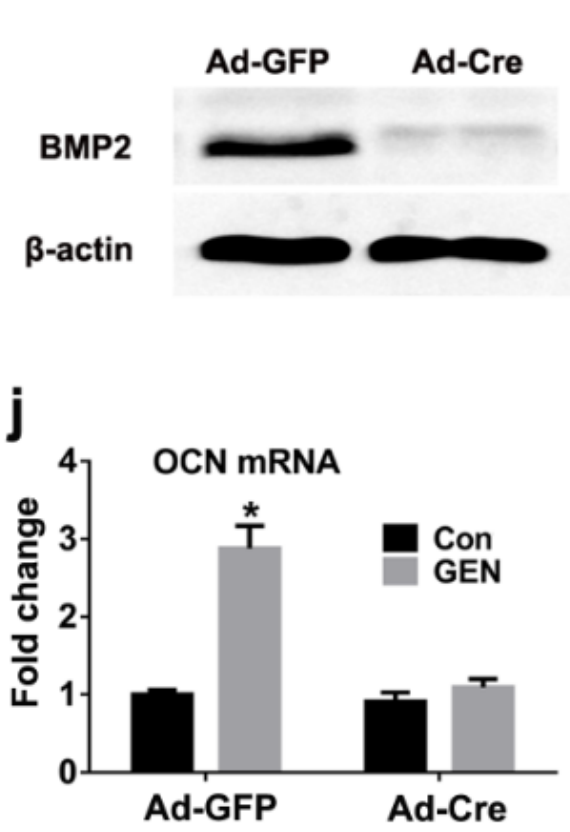

b

e

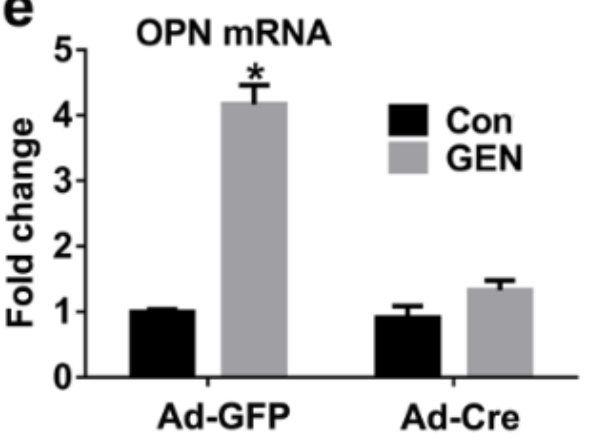

h

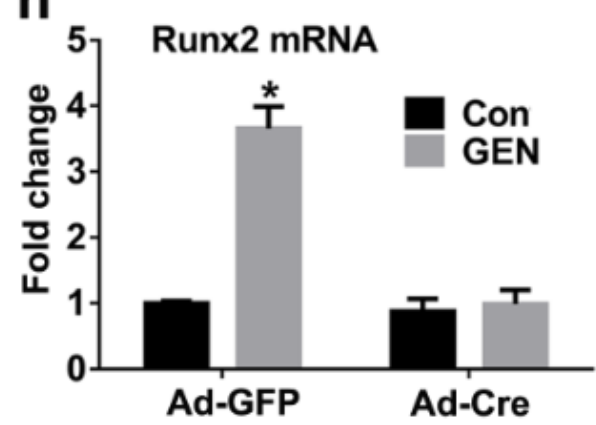

k

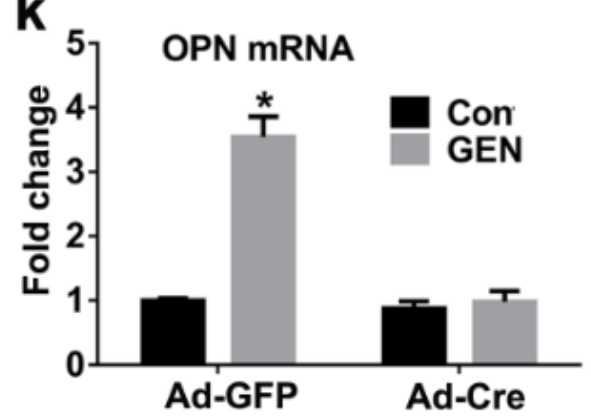

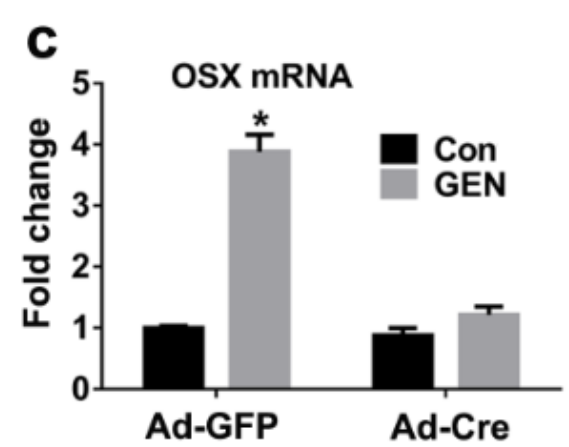

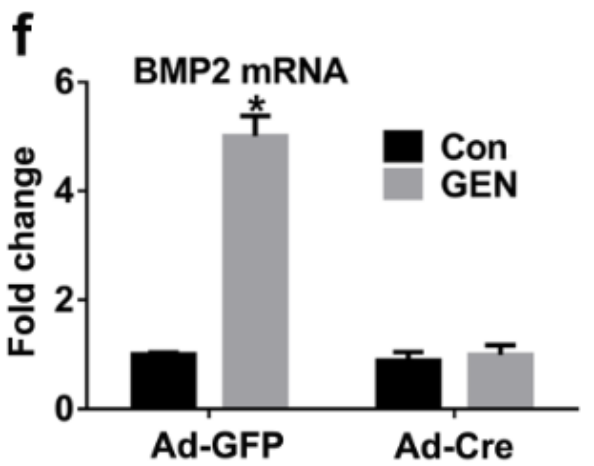

i

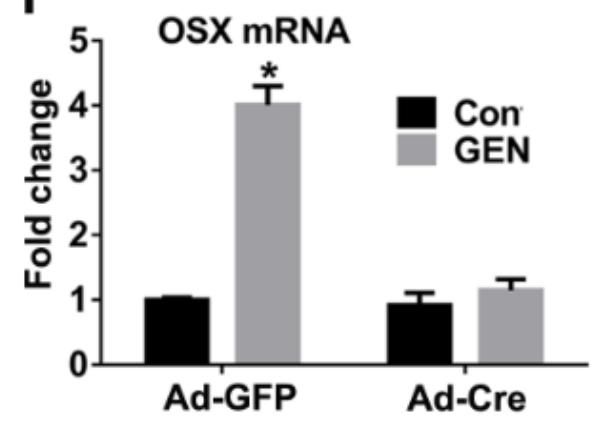

I

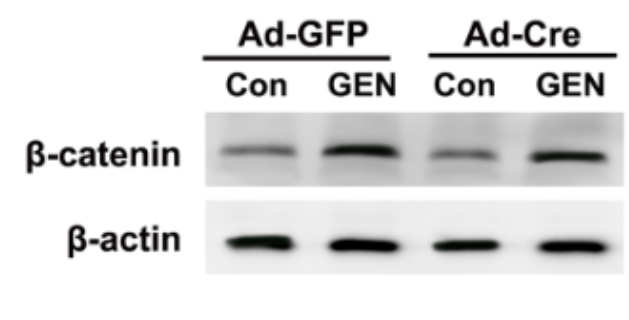

Figure 8

GEN promotes osteogenic differentiation of BMSCs in a $\beta$-catenin-BMP2 dependent manner. BMSCs were transfected with either Ad-GFP or Ad-Cre, which were used to delete the gene of $\beta$-catenin. Then the treated cells were incubated with GEN $(40 \mu \mathrm{M})$ for 7 days. The protein expression of $\beta$-catenin (a) was evaluated by western blot. The mRNA level of Runx2 (b), OSX (c), OCN (d), OPN (e), and BMP2 (f) were examined by q-PCR. BMSCs were transfected with either Ad-GFP or Ad-Cre, which were used to delete the 
gene of BMP2. Then the treated cells were incubated with GEN $(40 \mu \mathrm{M})$ for 7 days. The protein expression of BMP2 (g) and $\beta$-catenin (I) was estimated by western blot. The mRNA level of Runx2 (h), OSX (i), OCN (j), and OPN ( $k$ ) were tested by q-PCR. * $P<0.05$ compared with control (Con) group.

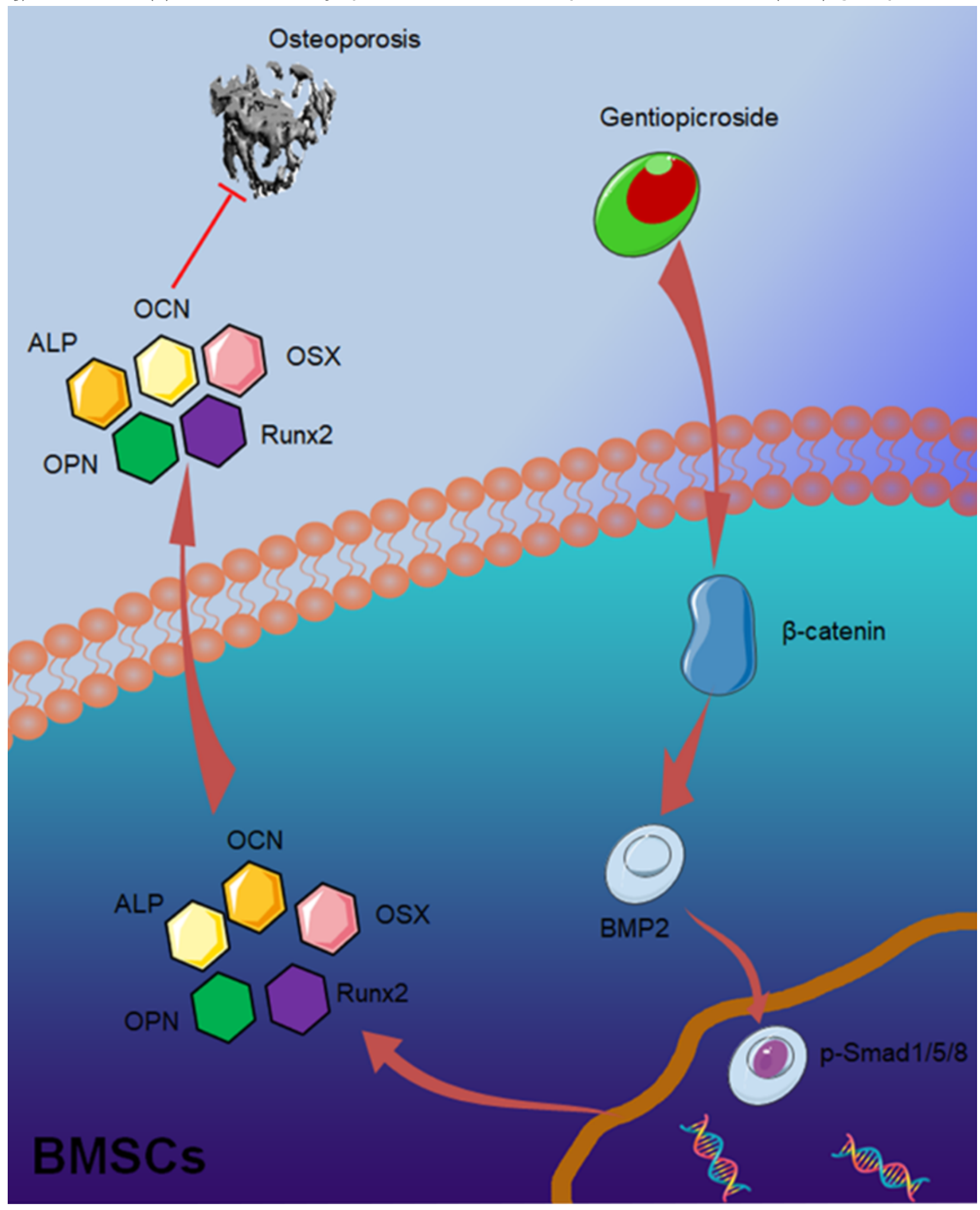

Figure 9

Proposed model depicting the underlying mechanisms of GEN in promoting the osteogenic differentiation of BMSCs 


\section{Supplementary Files}

This is a list of supplementary files associated with this preprint. Click to download.

- SupplementaryMaterial.docx 